El Guiniguada • Revista de investigaciones y experiencias en Ciencias de la Educación

\title{
AlUMNADO CON TDAH Y DEPORTES DE LUCHA
}

\section{STUDENTS WITH ADHD AND COMBAT SPORTS}

\author{
Rayco Montesdeoca Hernández \\ Guillermo Ruiz Llamas \\ Rosa Marchena Gómez \\ Jeremías Lasso Quintana
}

Universidad de Las Palmas de Gran Canaria

Recibido: 17/04/2015/Aceptado: 04/05/2015

RESUMEN

La presencia de alumnado con Trastorno por Déficit de Atención e Hiperactividad (TDAH) es más que evidente en nuestras aulas. Esto implica que se haga necesaria una intervención pedagógica adecuada por parte de maestros, profesores y familiares si perseguimos que estos chicos y chicas alcancen con éxito los objetivos de la educación obligatoria.

El presente artículo describe un estudio en el que trata de mostrar si el patrón comportamental de este tipo de alumnado puede ser compensado con los beneficios que genera la práctica de Deportes de Lucha. Para alcanzar este objetivo se diseñó una investigación que inicialmente midió, a través del test BASC (Behavior Assessment System for Children), los comportamientos que presentaban once sujetos diagnosticados con TDAH. Posteriormente se mantuvo con ellos ocho sesiones prácticas de Deportes de Lucha. Concluidas, se volvió a medir con el mismo instrumento de diagnóstico sus comportamientos. Los resultados evidencian que existen varios rasgos que han mejorado en estos sujetos (agresividad, conducta, habilidades sociales y autoestima) aunque se mantienen, con algunas variaciones, los restantes.

Palabras clave: Trastornos con Déficit de Atención e Hiperactividad (TDAH), Deportes de Lucha. 


\title{
Rayco Montesdeoca Hernández, Guillermo Ruiz Llamas, \\ Rosa Marchena Gómez, Jeremías Lasso Quintana
}

Alumnado con TDAH y deportes de lucha

\begin{abstract}
The presence of a students with Attention Deficit Hyperactivity Disorder (ADHD) is more than evident in our classrooms. This implies that a suitable pedagogic intervention is made necessary coming from teachers and families, if we want these boys and girls to reach with success the objectives of compulsory education.

This article describes a study in which the aim is to show if the behavioural pattern of this type of student body can be compensated with the benefits that generate practising Combat Sports. To reach this objective a research was designed that initially measured, through the BASC test (Behavior Assesment System for Children), the behaviours that presented eleven individuals diagnosed with ADHD. Subsequently eight sessions of Combat Sports were held with them, once being concluded, their behaviours were measured again with the same instrument. The results evidence that there are some aspects that have improved in these individuals (aggressiveness, behaviour, social abilities and self-esteem) although there were slight variations in others.
\end{abstract}

Keywords: Attention Deficit Hyperactivity Disorder (ADHD), Combat Sports.

\section{INTRODUCCIÓN}

El trastorno por déficit de atención con hiperactividad (TDAH), según el DSM5 de la American Psychiatric Association, presenta las siguientes características:

a) Un patrón persistente de inatención y/o hiperactividad-impulsividad que interfiere con el funcionamiento o el desarrollo

b) Algunos de esos síntomas están presentes antes de los 12 años del individuo

c) Los comportamientos derivados de estos rasgos se manifiestan en dos o más contextos, por ejemplo, en la casa, en la escuela y con los amigos o parientes

Esta inatención e hiperactividad-impulsividad interfiere con el funcionamiento social, académico o laboral, o reduce la calidad de los mismos. Estas interferencias son las que desencadenan los comportamientos sociales más censurados y criticados que se le dirige con frecuencia a este alumnado.

Este perfil descrito en el DSM-5 no siempre es idéntico en todos los individuos. En lo que se refiere a sus rasgos básicos según la American Psychiatric Association (2013), 


\section{Rayco Montesdeoca Hernández, Guillermo Ruiz Llamas, \\ Rosa Marchena Gómez, Jeremías Lasso Quintana \\ Alumnado con TDAH y deportes de lucha}

es posible encontrarnos una presentación combinada (inatención e hiperactividadimpulsividad), una presentación predominante con falta de atención sin cumplirse los otros síntomas o una presentación predominante hiperactiva/impulsiva.

Si estamos ante una presentación predominantemente con falta de atención, la persona puede presentar algunos de estos síntomas: parece no escuchar, tiene dificultades para seguir las instrucciones hasta el final, se organiza mal, evita o le disgustan las tareas que requieren un esfuerzo mental sostenido, pierde las cosas, es olvidadizo para las tareas diarias. Por otro lado, la presentación hiperactiva/impulsiva desencadenaría rasgos como los siguientes: mueve manos y pies en exceso, se levanta a menudo de su silla, interrumpe constantemente, es impaciente, habla sin parar, mordisquea los lápices o bolígrafos, también muerde la ropa, no piensa en las consecuencias de sus actos, no tiene percepción de peligro, dice lo primero que se le ocurre o tiene dificultades para dedicarse a tareas y juegos tranquilos. Y finalmente, el sujeto con una presentación combinada exhibiría síntomas conjuntos de las presentaciones antes mencionadas (Biederman y Faraone, 2000; Willcutt et alii, 2012; American Psychiatric Association, 2013).

A estas variantes hay que añadir que la afectación no siempre es idéntica en todos los sujetos. El número de síntomas que experimenta la persona y cuán dificultosos son estos síntomas en la vida cotidiana desencadena un gradiente en este trastorno que denominaríamos leve, moderado o grave.

\section{CONSECUENCIAS DEL TDAH}

Estudios como el de Barkley (2002) manifiestan que con frecuencia aparecen conductas agresivas o desafiantes (mentiras, pequeños robos, desafío a la autoridad), a medida que se acerca a la adolescencia. Entre un $20 \%$ y un $60 \%$ de estos niños y adolescentes, presentan conductas antisociales o trastorno disocial. Además pueden presentarse baja autoestima, tristeza, en algún caso depresión y desconfianza en el éxito futuro ya que muchos no terminan los estudios de Bachillerato y si lo hacen no suelen continuar con estudios universitarios. En el cuadro 1 se sintetiza de manera comparativa el porcentaje de aparición de determinados comportamientos entre la población joven con TDAH y sin TDAH. 


\section{Rayco Montesdeoca Hernández, Guillermo Ruiz Llamas, \\ Rosa Marchena Gómez, Jeremías Lasso Quintana \\ Alumnado con TDAH y deportes de lucha}

Cuadro 1. Resultados del estudio realizado por Barkley (2002)

\begin{tabular}{|l|l|l|}
\hline \multicolumn{3}{|c|}{ Resultados del estudio realizado por Barkley } \\
\hline Consecuencias & TDAH & Controles* \\
\hline Repiten curso & $42 \%$ & $13 \%$ \\
\hline Necesitaron apoyo escolar & $44 \%$ & $10 \%$ \\
\hline Acabaron la escuela & $68 \%$ & $100 \%$ \\
\hline Acceso a estudios superiores & $21 \%$ & $78 \%$ \\
\hline No terminan el Bachillerato & $31 \%$ & $10 \%$ \\
\hline Han tenido hijos antes de los 20 años & $26 \%$ & $1 \%$ \\
\hline $\begin{array}{l}\text { Han sido contagiados por enfermedades de } \\
\text { transmisión sexual }\end{array}$ & $17 \%$ & $4 \%$ \\
\hline No utilizaron métodos anticonceptivos & $25 \%$ & $10 \%$ \\
\hline
\end{tabular}

*Niños $\sin \mathrm{TDAH}$

\section{ESTRATEGIAS DE INTERVENCIÓN}

Los agentes implicados en la mejora de los menores con TDAH deben ser, por un lado, la escuela y la comunidad y por otro, de manera paralela, la familia, siendo este último contexto de gran importancia ya que de los padres va a depender en gran medida la evolución sintomatológica.

De manera sintetizada, a través de estos agentes intervinientes, existen tres líneas de actuación si buscamos la mejora de sus conductas: técnicas comportamentales o conductuales, técnicas cognitivas y fármacos. Las técnicas comportamentales se basarían en aumentar las conductas positivas haciendo uso de refuerzos positivos mediante sistemas de economía de fichas o similares. Las técnicas cognitivas basarían su actuación en ofrecer modelos de conducta reflexiva, enseñar a autoevaluar su comportamiento y en aprender autoinstrucciones o pasos para organizar el pensamiento.

Está evidenciado científicamente que las intervenciones conjuntas cognitivo-conductuales son eficaces en la mejora de estas personas (Presentación, Siegenthaler, Jara y Miranda, 2010). También el entrenamiento en habilidades sociales mejora el funcionamiento social y los niveles de asertividad.

Pero dado que estamos ante un trastorno que tiene un origen de base neurológica (American Psychiatric Association, 2013), existiendo una irregular producción de dopamina y noradrenalina, neurotransmisores que intervienen en las sinapsis neuronales, surgen los fármacos como una tercera línea de intervención y mejora. Aunque su uso es controvertido y presenta considerables efectos secundarios (ElSayed et alii, 2002), muchas de las personas diagnosticadas con TDAH (El-Sayed et 


\section{Rayco Montesdeoca Hernández, Guillermo Ruiz Llamas, \\ Rosa Marchena Gómez, Jeremías Lasso Quintana}

Alumnado con TDAH y deportes de lucha

alii, 2002), se encuentran medicadas con metilfenidato (neuroestimulante) o atomoxetina (no estimulante).

\section{LOS DEPORTES DE LUCHA}

Los deportes de lucha que desde el trabajo hemos puesto en práctica han sido la lucha canaria, kárate, judo y boxeo, así como diversos juegos de oposición. Hemos seleccionado los deportes de lucha canaria y judo ya que son deportes de contacto con agarre y por tanto los deportistas deben mantenerse en contacto en todo momento. Por otra parte decidimos utilizar otros dos deportes donde el contacto fuera intermitente, ya que en el karate y en el boxeo tienen que ir golpeándose.

Los Deportes de Lucha presentan beneficios normalmente desconocidos. Consideramos que su práctica por parte de chicos y chicas con TDHA puede permitirnos alcanzar objetivos como los siguientes (Carratalá y Carratalá, 2000; Hernández, 2000, Brocki et alii, 2010):

- Canalización de la hiperactividad.

- Adición de un elemento motivador e innovador que consiga centrar su atención.

- Desahogo de la conducta agresiva (si la presenta).

- Mejora de la autoestima.

- Mejora de la coordinación temporal y espacial.

- Desarrollo de la lateralidad.

Si tuviéramos que mencionar una justificación fisiológica para practicar deportes de lucha, sería generar de forma natural mayor cantidad de adrenalina y así compensar el déficit de dopamina y noradrenalina que presentan los sujetos con TDAH. De manera paralela, el carácter motivador de esta práctica deportiva puede aprovecharse como medio de enseñanza y aprendizaje capaz de corregir problemas de conducta (Reichenbach et alii, 1992).

Atendiendo a posibles comportamientos negativos presentes en los grupos con TDAH, hemos resumido en el cuadro 2 los beneficios que estos deportes de lucha podrían generar y compensar. 


\section{Rayco Montesdeoca Hernández, Guillermo Ruiz Llamas, Rosa Marchena Gómez, Jeremías Lasso Quintana \\ Alumnado con TDAH y deportes de lucha}

\section{Cuadro 2. Relación entre los comportamientos negativos del TDAH y los beneficios que desencadena la práctica de deportes de lucha}

\begin{tabular}{|l|l|}
\hline \multicolumn{2}{|l|}{$\begin{array}{l}\text { Relación entre los comportamientos negativos del TDAH y los beneficios que desencadena la } \\
\text { práctica de deportes de lucha }\end{array}$} \\
\hline Comportamientos & Beneficios \\
\hline Agresividad & $\begin{array}{l}\text { Canalizar la agresividad. Adquirir técnicas de } \\
\text { autocontrol. }\end{array}$ \\
\hline Poca tolerancia a la frustración & $\begin{array}{l}\text { Desarrollar la tolerancia por la derrota y aprender a } \\
\text { ganar }\end{array}$ \\
\hline Baja autoestima & Generar autoconfianza \\
\hline Hiperactividad & Adquirir técnicas de autocontrol \\
\hline $\begin{array}{l}\text { Falta de conocimiento sobre las consecuencias } \\
\text { negativas que pueden tener sus actos para si mismo y } \\
\text { para los demás. }\end{array}$ & $\begin{array}{l}\text { Establecer disciplina y valores como respeto y } \\
\text { honor. Trabajar las habilidades sociales }\end{array}$ \\
\hline Dificultades motrices & Mejorar la coordinación. \\
\hline
\end{tabular}

\section{Método}

El objetivo de este estudio fue averiguar si los deportes de lucha aportan beneficios al alumnado con TDAH. Para alcanzar este propósito se seleccionó una muestra de once sujetos que se dividieron en dos grupos. Uno de ellos - grupo A- tenía cinco sujetos en edades comprendidas entre 13 y 18 años con tres chicos y dos chicas. El segundo - grupo B- estaba compuesto por seis sujetos entre 7 y 10 años con cinco chicos y una chica.

Todos ellos estaban diagnosticados con TDAH y, en el caso del grupo B, tal como se muestra en el cuadro 3, estaban todos sometidos a un tratamiento farmacológico. En cambio, en el grupo A encontramos dos sujetos, los que tenían más edad, sin medicar. El porcentaje, por tanto, de sujetos medicados en el grupo B era del 100\%, mientras que en el grupo A del 60\%.

\section{Cuadro 3. Clasificación de los sujetos en función de la edad, el tratamiento con fármacos y los grupos de pertenencia}

\begin{tabular}{|l|l|}
\hline B & A \\
\hline 1.Medicado (7 años) & 1. Medicada (13 años) \\
\hline 2. Medicada (9 años) & 2. Medicada (15 años) \\
\hline 3. Medicado (9 años) & 3. Medicado (16 años) \\
\hline 4. Medicado (10 años) & 4. No medicado (17 años) \\
\hline 5. Medicado (10 años) & 5. No medicado (18 años) \\
\hline 6. Medicado (10 años) &
\end{tabular}




\section{Rayco Montesdeoca Hernández, Guillermo Ruiz Llamas, \\ Rosa Marchena Gómez, Jeremías Lasso Quintana \\ Alumnado con TDAH y deportes de lucha}

\section{Características del diseño}

En una fase previa se recogió información sobre las características de los chicos y chicas pertenecientes a la muestra. Para ello se utilizaron las siguientes técnicas:

- Observación previa de los participantes en ambientes espontáneos con la finalidad de recoger información sobre sus comportamientos

- Entrevistas con los padres, tanto en esta fase previa como de manera posterior al desarrollo de las sesiones deportivas.

- Análisis de las calificaciones académicas aportadas por los padres

- Cumplimentación del Test BASC (Sistema de Evaluación de la Conducta de Niños y Adolescentes).

Este test -BASC (Behavior Assessment System for Children)- permite evaluar los aspectos adaptativos e inadaptativos de la conducta de niños y adolescentes. Ofrece la posibilidad de recoger esa información tanto de los padres y profesores como del propio sujeto. Además, añade a estas fuentes de información un sistema de observación del sujeto y un formato de historia clínica (Reynolds y Kamphaus, 1992). Puede ser aplicado de modo conjunto o por separado, como ha sido el caso. Incluye tanto la evaluación de los aspectos positivos (liderazgo, habilidades sociales, autoestima...) como los negativos (ansiedad, agresividad, problemas de atención, problemas de aprendizaje, hiperactividad, retraimiento...). Es una de las pruebas más reconocidas internacionalmente y constituye una herramienta esencial para evaluar el nivel de adaptación de la conducta de los niños y los adolescentes, tanto en el campo escolar como en el clínico.

Una vez evaluados los sujetos mediante las técnicas descritas, se obtuvo una información y unos parámetros de comportamiento de gran interés para tenerlos presentes en la fase de desarrollo e intervención con los sujetos. Pusimos en práctica diferentes sesiones de actividad física, un total de ocho, en las que se practicaron deportes como lucha canaria, kárate, judo y boxeo entre otros, así como variedad de juegos de oposición. El lugar donde se desarrollaron estas actividades de deporte fue siempre el mismo, un espacio habilitado con todo lo necesario en el gabinete colaborador del proyecto. Éste se encuentra en el municipio de Santa Lucía en la isla de Gran Canaria.

En todas las sesiones que se tuvieron se contó con un tatami y un reproductor musical. Los materiales utilizados, tanto colectivos como individuales, están descritos en el cuadro 4. 


\section{Rayco Montesdeoca Hernández, Guillermo Ruiz Llamas, \\ Rosa Marchena Gómez, Jeremías Lasso Quintana \\ Alumnado con TDAH y deportes de lucha}

\section{Cuadro 4. Clasificación de los recursos materiales en colectivos e individuales}

\begin{tabular}{|l|l|}
\hline Colectivos & Individuales \\
\hline Barras de goma espuma ("churros") & Ropa cómoda \\
\hline Pelotas de espuma & Guantes de boxeo/"guantillas" de Kárate \\
\hline Aros & \\
\cline { 1 - 1 } Cuerdas & \\
\cline { 1 - 1 } Conos & \\
\cline { 1 - 1 } Palos de golpeo & \\
\cline { 1 - 2 } Sacos de golpeo &
\end{tabular}

En la última fase -fase final- se evalúa nuevamente a los sujetos de la muestra con el test BASC y se vuelve a entrevistar a los padres. La finalidad es obtener información sobre los comportamientos que mostraban una vez que habían practicado sesiones de Deportes de Lucha. Con los resultados obtenidos se establecen unas conclusiones y se elabora el informe de la investigación realizada. El cuadro 5 se refiere de manera detallada el cronograma que establecimos y la temporalización de cada una de las fases que explicadas.

\section{Resultados}

Para organizar los resultados obtenidos, hemos elaborado el cuadro 6 donde se muestran los datos e informaciones recogidas tanto en la fase previa como en la final del proyecto. Concretamente, aparece la siguiente información:

Grupos a los que pertenecen los diferentes sujetos.

Parámetros elegidos para la intervención aportados por los resultados de los test y la entrevista previa con los padres. Estos parámetros son: agresividad, hiperactividad, problemas de atención, problemas de conducta, baja autoestima y ausencia de habilidades sociales.

Evaluación previa y posterior del sujeto. Los datos e informaciones recogidos de forma previa a la intervención indican si el sujeto muestra el problema de conducta o comportamiento mientras que, los datos e informaciones recogidos posteriormente a la intervención, nos indican si el sujeto ha mejorado o no en dicho parámetro.

De manera gráfica, podemos también observar los resultados obtenidos en porcentajes por cada grupo en la situación inicial y posterior al desarrollo de las sesiones de Deportes de Lucha (gráficas 1 y 2). 
Rayco Montesdeoca Hernández, Guillermo Ruiz Llamas,

Rosa Marchena Gómez, Jeremías Lasso Quintana

Alumnado con TDAH y deportes de lucha

Cuadro 5. Cronograma de las fases y los tiempos del proyecto

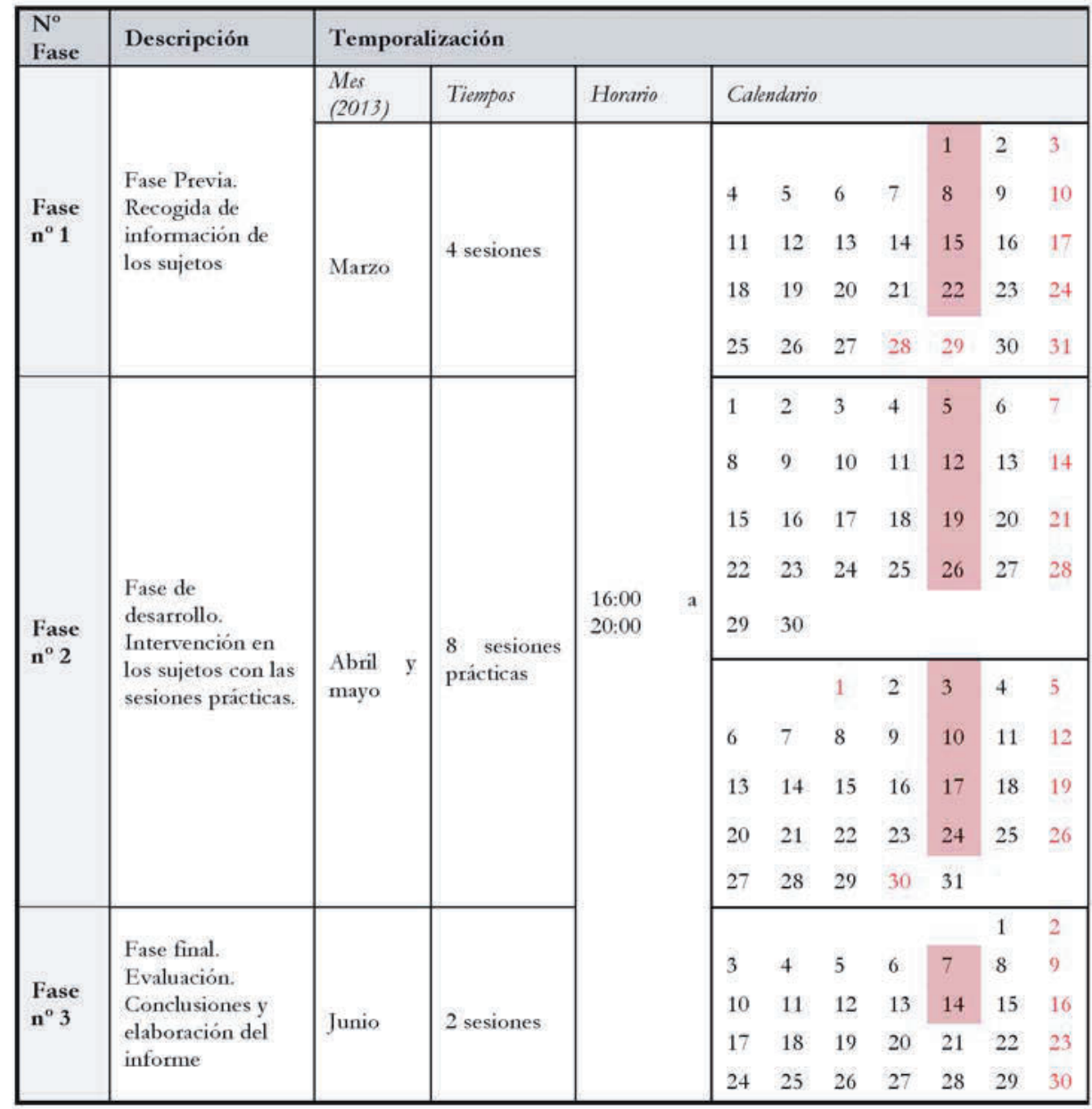


Rayco Montesdeoca Hernández, Guillermo Ruiz Llamas,

Rosa Marchena Gómez, Jeremías Lasso Quintana

Alumnado con TDAH y deportes de lucha

Cuadro 4.1. Información obtenida antes y después de la intervención

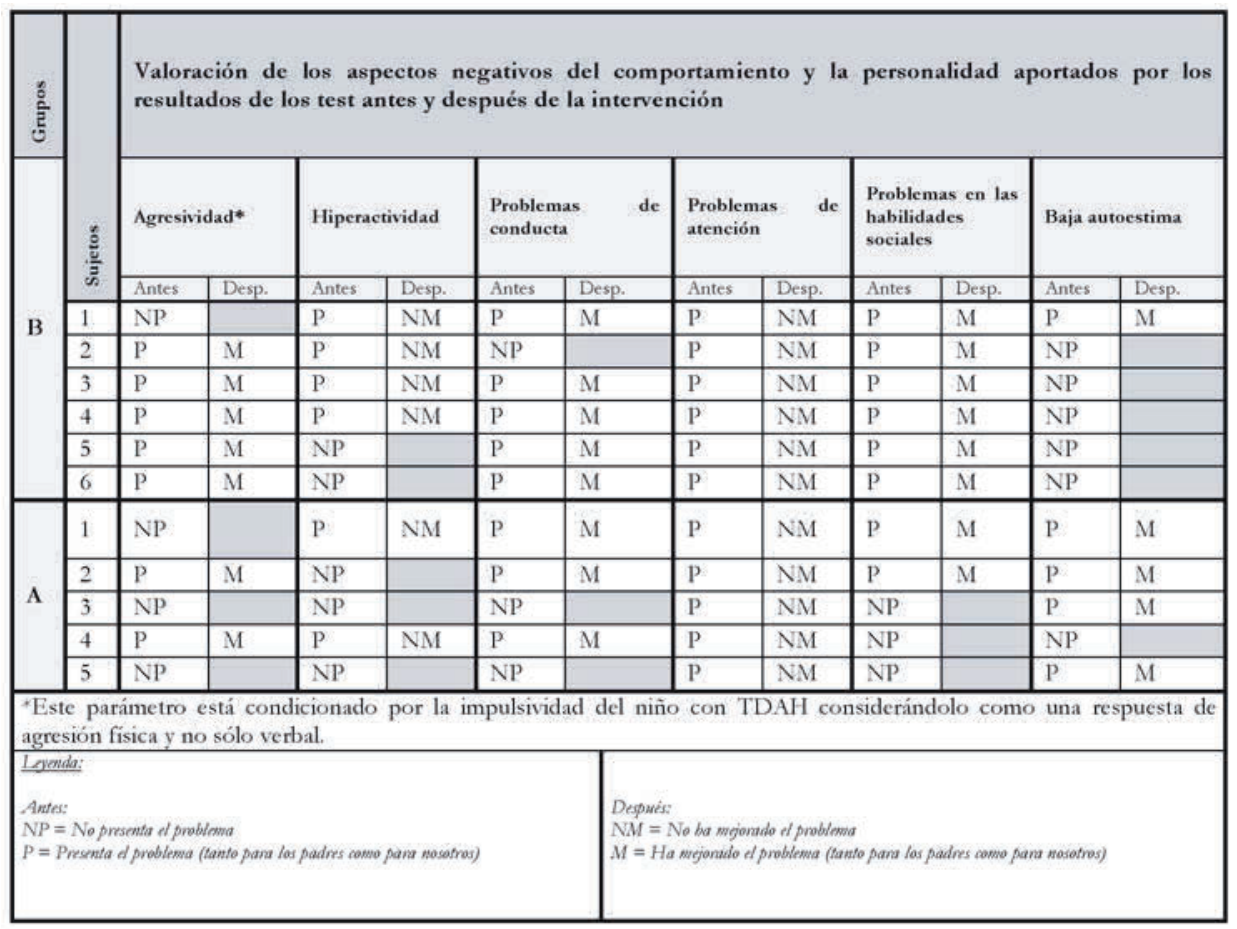

Gráfica 1. Relación entre la situación inicial y posterior de los sujetos pertenecientes al grupo B

\begin{tabular}{|l|c|c|c|c|c|c|c|c|}
\hline \multicolumn{7}{c|}{ Grupo B } & \\
\hline
\end{tabular}


Rayco Montesdeoca Hernández, Guillermo Ruiz Llamas,

Rosa Marchena Gómez, Jeremías Lasso Quintana

Alumnado con TDAH y deportes de lucha

\section{Gráfica 2. Relación entre la situación inicial y posterior de los sujetos pertenecientes al grupo A}

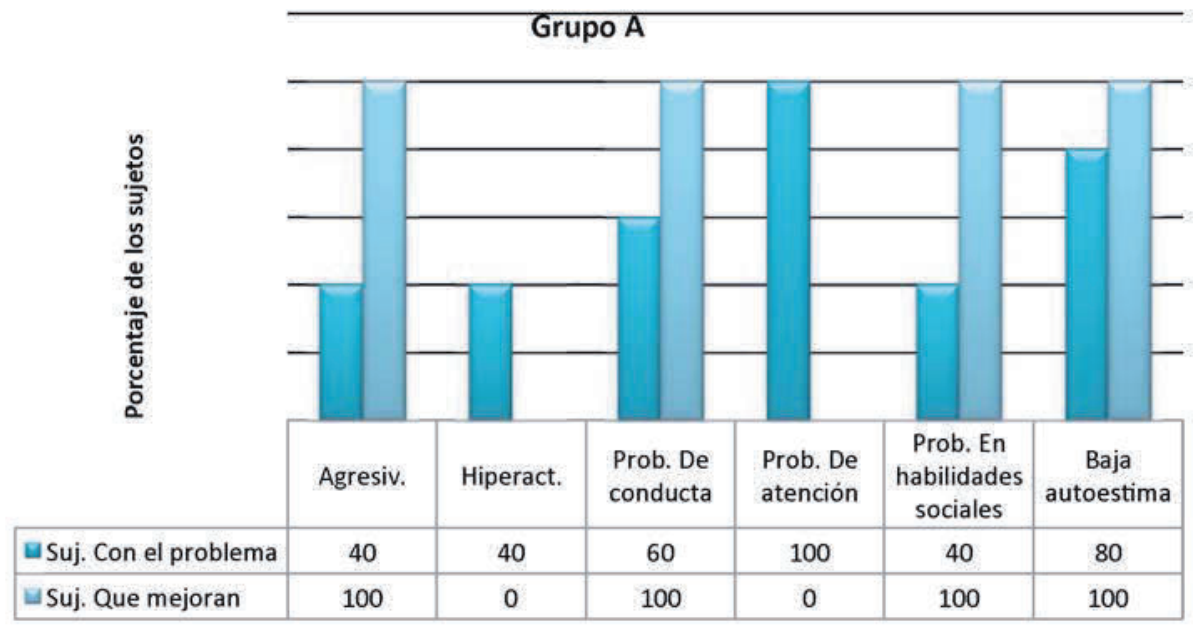

\section{Discusión}

Podemos afirmar que, en general, los resultados han sido positivos si tenemos en cuenta que, después de que los chicos y chicas participaran en diferentes sesiones de deportes de lucha, ha habido una mejora en diversos aspectos de sus comportamientos. Aunque es cierto que, dado los aspectos que han mejorado (agresividad, problemas de conducta, habilidades sociales y autoestima) cualquier otro deporte de colaboración y/o oposición podría haber desencadenado los mismos avances, dadas las tareas conjuntas y colaborativas que deben practicarse (Brocki et alii, 2010; Dustin et alii, 2015). También habría que considerar en el estudio, variable que no hemos controlado en esta ocasión, el tipo de interacción que se estableció entre los entrenadores y los sujetos de la muestra. La presencia de estrategias conductuales o cognitivas en la relación social que mantuviesen, sabemos que podrían haber producido un incremento de las conductas positivas en los sujetos con TDAH (Lavigueur, 2002).

Sorprende desde el estudio que en hiperactividad y problemas de atención -sintomatología nuclear que define el TDAH- no haya existido mejoría en ninguno de los dos grupos de trabajo.

Se observó, no obstante, que durante la práctica de los deportes, sumergidos en los elementos lúdicos que perseguían la motivación de los sujetos, conseguíamos paliar el déficit de atención característico y frenar, de manera considerable, la hiperactividad. 


\section{Rayco Montesdeoca Hernández, Guillermo Ruiz Llamas, \\ Rosa Marchena Gómez, Jeremías Lasso Quintana \\ Alumnado con TDAH y deportes de lucha}

Finalizadas las sesiones, sin embargo, desaparecían estos comportamientos de avance. Es posible pensar que estas conductas mejoradas, para que se generalizaran en otras situaciones y no solo emergieran mientras se desarrollaba la sesión deportiva, sea preciso un trabajo más a largo plazo. Posiblemente las sesiones de intervención que se aplicaron desde nuestra investigación hayan sido escasas para alcanzar ese fin.

Otro tema de discusión hace referencia a los sujetos que muestran aspectos de agresividad, hiperactividad, problemas de conducta y de habilidades sociales que disminuyen en el grupo de A frente a los del grupo de B. Es posible que con la edad se vayan compensando déficits relacionados con el autocontrol y el desarrollo de la personalidad.

Por otra parte la baja autoestima está más elevada en el grupo A. Pensamos que debido al mayor desarrollo físico, pueden aumentar los complejos por el autoconcepto y su preocupación por cómo lo ven los demás. Así mismo tenemos que decir que la baja autoestima ha sido el parámetro más destacable en cuanto a mejoría en el grupo de A una vez acabada la intervención (Ruales y Cepeda, 2012).

En cuanto a la variable de género entre los sujetos, ésta ha sido insuficiente como para poder comparar resultados. En nuestra muestra se cumplió la advertencia constatada de que la frecuencia del TDAH es mucho más alta en varones que en mujeres.

Respecto a la variable que hace referencia a los sujetos no medicados, respecto a los sí medicados, no son suficientes como para hacer comparaciones entre los resultados, pero es importante conocer que en estos sujetos, aunque ha habido mejoría, ésta ha sido mucho más lenta que en los demás sujetos.

\section{ConClusiones}

Las conclusiones de esta investigación podemos resumirlas en los siguientes puntos:

- Los práctica de diferentes deportes de lucha y juegos de oposición mejoran aspectos negativos de la conducta y la personalidad en niños y adolescentes con TDAH, aunque sería conveniente analizar las características de la interacción establecida entre los entrenadores y los sujetos en el momento de en que se desarrollan estas prácticas dada la importancia que poseen en el avance comportamental de las personas con TDAH. (Brocki, 2010; Dustin et alii, 2015)

- Los aspectos que mejoran son agresividad, problemas de conducta, problemas en habilidades sociales y autoestima. (Lavigueur, 2002; Ruales y Cepeda, C., 2012) 


\section{Rayco Montesdeoca Hernández, Guillermo Ruiz Llamas, \\ Rosa Marchena Gómez, Jeremías Lasso Quintana}

Alumnado con TDAH y deportes de lucha

- A mayor edad son menos frecuentes los problemas relacionados con la conducta como agresividad, hiperactividad, problemas de conducta y problemas en habilidades sociales. (Lavigueur, 2002; Ruales y Cepeda, 2012)

- A mayor edad es más frecuente la baja autoestima, problema relacionado con la personalidad. (Lavigueur, 2002; Ruales y Cepeda, 2012)

- En los sujetos no medicados, aunque han sido escasos desde nuestra muestra, el grado de mejoría ha sido menor.

- La hiperactividad y los problemas de atención han mejorado sólo durante la propia actividad física y durante un breve tiempo posterior a las sesiones prácticas.

\section{BibLIOGRAFÍA}

American Psychiatric Association. (2013). Diagnostic and Statistical Manual of Mental Disorders (DSM- V). Washington, D.C: Masson.

Barkley, R. A. (2002). "Psychosocial treatment for Attention Deficit Hiperactivity Disorder (ADHD) in children". Clinic Psychiatry,63, 36-43.

Biederman, J.,Mick, E., y Faraone, S. V. (2000). "Age-dependent decline of symptoms of ADHD: impact of remission definition and symptom type". American Journal of Psychiatry, 157, 816-818.

Brocki, K. C., Tillman, C. M., \& Bohlin, G. (2010). "CPT performance, motor activity, and continuous relations to ADHD symptom domains: a developmental study". The European Journal of Developmental Psychology, 7, 178-197.

Carratalá, V. y Carratalá, E. (2000). Judo: la actividad física y deportiva extraescolar en los centros educativos. Madrid: Consejo Superior de Deportes.

Dustin E., Mark D., Michael J., Joseph S., y Lauren M. (2015). "Hyperactivity in AttentionDeficit/Hyperactivity Disorder (ADHD): Impairing Deficit or Compensatory Behavior?” Journal of Abnormal Child Psychology, Online ISSN 1573-2835.

El-Sayed, E., Larsson, J. O., Persson, H. E., y Rydelius, P. A. (2002). "Altered cortical activity in children with attention-deficit/hyperactivity disorder during attentional load task". Journal of the American Academy of Child and Adolescent Psychiatry, 41, 811-819.

Hernández, J. (2000). La iniciación a los deportes desde su estructura y dinámica: Aplicación a la Educación Física Escolar y al Entrenamiento Deportivo. Barcelona: INDE.

Lavigueur, S. 2002. «Ces parents à bout de souffle; Un guide de survie ». Les éditions Quebecor. «Une entrevue avec le docteur Claude Desjardins, pédiatre ». Outremont (Québec). p. 231 et suivantes, 379 et suivantes.

Presentación, M. J., Siegenthaler, R., Jara, P. y Miranda, A. (2010). "Seguimiento de los efectos de una intervención psicosocial sobre la adaptación académica, emocional y social de niños con TDAH". Revista de Neurología, 22, 778-783.

Reichenbach, L. C., Halperin, J. M., Sharma, V., y Newcorn, J. H. (1992). “Children's motor activity: reliability and relationship to attention and behavior". Developmental Neuropsychology, 8, 87-97. 


\section{Rayco Montesdeoca Hernández, Guillermo Ruiz Llamas, \\ Rosa Marchena Gómez, Jeremías Lasso Quintana \\ Alumnado con TDAH y deportes de lucha}

Reynolds, C. R., y Kamphaus, R. W. (1992). Behavior Assessment System for Children (BASC). Minnesota: TEA Ediciones.

Ruales, V. M., y Cepeda, C. (2012). Efectividad en la terapia cognitivo conductual con técnicas basadas en el condicionamiento operante para el mejoramiento de autoestima en niños con TDAH. Quito: UCE.

Willcutt, E. G., Nigg, J. T., Pennington, B. F., Carlson, C. L., McBurnett, K., Rohde, L. A., y Lahey, B. B. (2012). "Validity of DSM-IVattention-deficit/hyperactivity disorder symptom dimensions and subtypes". Journal of Abnormal Psychology, 121, 991-1010.

rayco.montesdeoca@ulpgc.es / guillermo.ruiz@ulpgc.es rosa.marchena@ulpgc.es / jere.lasso@activaeduca.com 